\title{
ERRATUM
}

\section{Erratum to: Mechanical performance and dimensional stability of nano-silver impregnated densified spruce wood}

\author{
Ghonche Rassam • Mohammad Ghofrani . \\ Hamid Reza Taghiyari • Behnam Jamnani • \\ Mohamad Ali Khajeh
}

Published online: 19 January 2012

(C) Springer-Verlag 2012

Erratum to: Eur. J. Wood Prod.

DOI 10.1007/s00107-011-0590-7

Unfortunately, Tables 1 and 2 in the published version of this article contained mistakes. The corrected tables are printed below.

Table 1 Specifications* of nano-silver solution

Tab. 1 Eigenschaften der Nanosilber-Lösung

\begin{tabular}{lllll}
\hline Material & Concentration $[\mathrm{ppm}]$ & $\mathrm{Ag}$ particles diameters $[\mathrm{nm}]$ & Reduction agent $^{* *}$ & Absorption $^{* * *}\left[\mathrm{~g} / \mathrm{cm}^{3}\right]$ \\
\hline $\begin{array}{l}\mathrm{AgNO}_{3} \& \text { Pure } \\
\text { De-ionized water }\end{array}$ & 200 & $10-100$ & $\mathrm{NaBH}_{4}$ & 0.12 \\
\hline
\end{tabular}

*From Jafar Sorkh Company

*** Releases Ag ions in solution

*** $\mathrm{g}$ absorbed $\mathrm{AgNO}_{3}$ solution per each $\mathrm{cm}^{3}$ wood

The online version of the original article can be found under doi:10.1007/s00107-011-0590-7.

G. Rassam (凶) · M. Ghofrani · H.R. Taghiyari · M.A. Khajeh

Department of Wood Science and Technology, Faculty of Civil

Engineering, Shahid Rajaee Teacher Training University, Tehran,

Iran

e-mail: ghrassam@yahoo.com

Table 2 Density of all densified specimens under different press conditions

Tab. 2 Dichte der Pressholzprüfkörper in Abhängigkeit der Pressbedingungen

\begin{tabular}{lccll}
\hline Specimen No. & $\begin{array}{l}\text { Time } \\
{[\mathrm{h}]}\end{array}$ & Temperature & $\begin{array}{l}\text { Treatment } \\
{\left[{ }^{\circ} \mathrm{C}\right]}\end{array}$ & $\begin{array}{l}\text { Density } \\
{\left[\mathrm{g} / \mathrm{cm}^{3}\right]}\end{array}$ \\
\hline 1 & & & Dry & 0.47 \\
2 & & 150 & Water & 0.47 \\
3 & 1 & & Nano-silver & 0.47 \\
4 & & & Dry & 0.48 \\
5 & & Water & 0.48 \\
6 & & & Nano-silver & 0.49 \\
7 & & Dry & 0.50 \\
8 & & & Water & 0.47 \\
9 & 450 & & Nano-silver & 0.51 \\
10 & & Dry & 0.51 \\
11 & & & Water & 0.47 \\
12 & & & Nano-silver & 0.53 \\
Control & & & & 0.36 \\
\hline
\end{tabular}

B. Jamnani

Graduated Engineer in Natural Resources, Wood and Paper

Science and Technology, Tehran University, Tehran, Iran 